3. Bowser D, Hill K. Exploring evidence for disrespect and abuse in facility-based childbirth: Report of a Landscape analysis [Internet]. Boston: USAID-TRAction Project Harvard School of Public Health; 2010 [citado el 22 de mayo del 2015]; Disponible en: http://www.tractionproject. org/resources/access-skilled-care-respectful-maternal-care/ exploring-evidence-disrespect-and-abuse

4. Comité de América Latina y el Caribe para la Defensa de los Derechos de la Mujer. Silencio y complicidad. Violencia contra las mujeres en los servicios públicos de salud del Perú. Lima: CLADEM/CRLP; 1998.

5. Windau-Melmer T. A guide for advocating for respectful maternity care. Washington DC: USAID; 2013.

Correspondencia: María Reneé Montesinos-Segura

Dirección: Urbanización Marcavalle A-20 Wanchaq, Cusco, Perú Teléfono: 984174057

Correo electrónico:mrenee.ms@gmail.com

\section{EDUARDO PRETELL Y LA LUCHA CONTRA LA DEFICIENCIA DE YODO EN EL PERÚ}

\section{EDUARDO PRETELL AND THE FIGHT AGAINST IODINE DEFICIENCY IN PERU}

\author{
Gustavo F. Gonzales ${ }^{1, a}$, Jaime E. Villena ${ }^{2, b}$
}

Sr. Editor. En el último número de su revista aparece el artículo "Concentraciones de yodo en orina y en sal de consumo en mujeres entre 12 a 49 años del Perú", cuyos autores son del Instituto Nacional de Salud. El estudio es importante, pues confirma la eliminación de la deficiencia de yodo en el Perú y sugiere la posibilidad de una sobreingesta de yodo, en base a la encuesta realizada en una población de 4188 mujeres, en la que la mediana de yodo en orina se encuentra por encima de lo recomendado por la OMS. Llama la atención, sin embargo, que en dicho artículo se ignore los estudios previos sobre este tema llevados a cabo en el Perú $y$, de manera particular, que no haga mención a las publicaciones del Dr. Eduardo Pretell, pionero en los estudios experimentales y clínicos sobre la deficiencia de yodo y artífice de la política y el programa para la

\footnotetext{
1 Instituto de Investigaciones de la Altura, Facultad de Ciencias y Filosofía, Universidad Peruana Cayetano Heredia. Lima, Perú.

2 Instituto de Investigaciones de la Altura y Facultad de Medicina, Universidad Peruana Cayetano Heredia. Lima, Perú.

a Doctor en Medicina y doctor en Ciencias; ${ }^{\text {b }}$ doctor en Medicina Recibido: 30-07-15 Aprobado: 05-08-15
}

Citar como: Gonzales GF, Villena JE. Eduardo Pretell y la lucha contra la deficiencia de yodo en el Perú [carta]. Rev Peru Med Exp Salud Publica. 2015;32(3):610-11. erradicación de los desórdenes por deficiencia de yodo en el Perú y en América Latina. El Dr. Pretell fue el gestor, fundador y director por 10 años del programa nacional para la eliminación de esta deficiencia, gracias al cual el Perú es el primer país en logar su erradicación de manera sostenida, éxito reconocido por los organismos internacionales.

Desde 1966, Eduardo Pretell, y los colaboradores del Instituto de Investigaciones de la Altura de la Universidad Peruana Cayetano Heredia, reinició la investigación de la deficiencia de yodo en el Perú y, en paralelo con estudios epidemiológicos, confirmaron la persistencia y severidad de la deficiencia de yodo, del bocio y del cretinismo en vastas regiones de Perú (1). Por otro lado, Pretell ha estudiado el impacto de la deficiencia de yodo durante la gestación y su impacto dañino en la madre, pero sobre todo en el feto ${ }^{(2)}$. Así mismo, ha investigado el beneficio del uso del aceite yodado como un nuevo método alternativo para la prevención y tratamiento de esta deficiencia ${ }^{(3)}$, el cual ha sido recomendado por la OMS y es usado en muchos países donde el consumo de sal yodada no está aún asegurado.

Los resultados de estas investigaciones sirvieron de base para que el Ministerio de Salud, en 1983, creara la "Oficina de Bocio Endémico" (D.S. 047-83$\mathrm{SA}$ ), posteriormente denominado "Programa Nacional de Control del Bocio y Cretinismo Endémicos", (PRONABCE) en 1987, y más tarde "Programa Nacional de Erradicación de los Desórdenes por Deficiencia de lodo". Este programa ha sido reconocido como uno de los programas más exitosos a nivel internacional. Una publicación en el 2008 por Pretell e Higa da testimonio de este éxito ${ }^{(4)}$

Su labor se ha extendido a nivel regional con logros extraordinarios. En el 2004, con el programa Thyromobil, estudió 163 sitios en 13 países e hizo seguimiento de yodo urinario en 8208 muestras describiendo la presencia de exceso de yodo en varios lugares evaluados $\mathrm{y}$, recientemente, ha evaluado la situación actual en todos los países de Latinoamérica, demostrando que en prácticamente toda la región se ha logrado la eliminación de los desórdenes por deficiencia de yodo ${ }^{(5)}$.

El liderazgo del Dr. Pretell ha sido reconocido internacionalmente, en el año 2006 recibió el premio Reina Sofía por su eficiente gestión en Perú y en el 2009 el premio Abraham Horwitz otorgado por la Organización Panamericana de la Salud y PAHF por su extraordinaria contribución para la eliminación de la deficiencia de yodo en las Américas. Es fundador del International Council for Control of lodine Deficiency Disorders (ICCIDD) y coordinador regional para 
América en desórdenes por deficiencia de yodo. Es importante señalar a las nuevas generaciones la contribución científica y epidemiológica de pioneros como el Dr. Eduardo Pretell y que estos hallazgos no solamente hayan quedado en publicaciones, sino que se han traducido en la implementación de las medidas apropiadas que han impactado en la salud pública de nuestro país y de la región con el mejoramiento la calidad de vida de nuestros compatriotas.

Fuente de financiamiento: autofinanciado.

Conflictos de interés: los autores declaran no tener conflictos de interés en la publicación de este artículo.

\section{REFERENCIAS BIBLIOGRAFICAS}

1. Pretell EA. Desórdenes por deficiencia de yodo (DDI). Generalidades y situación en el Perú. En: Blanco de Alvarado T, Gonzales Mugaburu L, ed. Situación nutricional en el Perú. Lima: MINSA-OPS; 1989. p. 395-451.

2. Pretell EA, Cáceres A. Impairment of mental development by iodine deficiency and correction. En: Stanbury JB. The Damaged Brain of Iodine Deficiency: Cognitive, Behavioral, Neuromotor, Educative Aspects. New York: Cognizant Communication Corporation; 1994. p. 187-93.

3. Pretell EA, Moncloa F, Salinas R, Kawano A, Guerra-Garcia R, Gutierrez L, et al. Prophylaxis and treatment of endemic goiter in Peru with iodized oil. J Clin Endocrinol Metab. 1969;29(12):1586-95.

4. Pretell EA, Higa AM. Eliminación sostenida de los desórdenes por deficiencia de yodo en Perú: 25 años de experiencia. Acta Med Peruana. 2008;25(3):128-34.

5. Pretell EA, Eliminación sostenible de los desórdenes por deficiencia de yodo en Latinoamérica. Lima: Iodine Global Network-UNICEF-OPS; 2014.

Correspondencia: Gustavo Francisco Gonzales Rengifo

Dirección: Av. San Borja Norte 1234

Teléfono: (+511) 3190000 anexo 2535

Correo electrónico: gustavo.gonzales@upch.pe

\section{COMENTARIOS AL ARTÍCULO: CONCENTRACIONES DE YODO EN ORINA Y EN SAL DE CONSUMO EN MUJERES ENTRE 12 A 49 AÑOS DEL PERÚ}

\author{
COMMENTS TO THE ARTICLE: IODINE \\ CONCENTRATIONS IN URINE AND SALT \\ CONSUMPTION IN WOMEN BETWEEN THE \\ AGES OF 12 TO 49 IN PERU
}

\author{
Eduardo A. Pretell1,a
}

Sr. Editor. El artículo es importante en cuanto confirma la eliminación de la deficiencia de yodo como problema de salud pública en nuestro país, y demuestra que el Centro Nacional de Alimentación y Nutrición (CENAN) del Instituto Nacional de Salud (INS), mantiene la vigilancia de la calidad de la sal yodada y de la nutrición de yodo, función que le ha sido encomendada en reemplazo del desaparecido Programa Nacional de Erradicación de los Desórdenes por Deficiencia de Yodo (PNEDDI) del Ministerio de Salud, y cuyo cumplimiento es vital para la eliminación sostenible de estos desórdenes, la cual ha sido lograda en nuestro país a través de un plan estratégico modelo y el importante apoyo político del Ministerio de Salud (MINSA) ${ }^{(1)}$.

Considero conveniente, sin embargo, hacer algunos comentarios y precisiones sobre los datos proporcionados por los autores del artículo, todos ellos pertenecientes al personal profesional del CENAN.

La mediana de la concentración urinaria de yodo (CUI) es reconocida como el mejor indicador de la nutrición e ingesta de yodo. La mediana de 250,4 $\mu \mathrm{g} / \mathrm{L}$ reportada en el artículo, en un grupo de 4188 mujeres de 12 a 49 años de edad procedentes de diferentes regiones del país, si bien, esta cifra está sobre el rango señalado hace ocho años y referido como adecuado por la OMS-UNICEF-ICCIDD, no llega al nivel de excesivo $(\geq 300 \mu \mathrm{g} / \mathrm{L})$ y cae más bien en el rango de $200-299 \mu \mathrm{g} / \mathrm{L}$, lo que permite asegurar una mayor ingesta de yodo requerida durante la gestación

\footnotetext{
1 Universidad Peruana Cayetano Heredia. Lima, Perú

a Coordinador Regional para América del Iodine Global Network, fundador y exdirector del desaparecido Programa Para la Eliminación de los Desórdenes por Deficiencia de Yodo del Ministerio de Salud. Recibido: 25-07-15 Aprobado: 05-08-15
}

Citar como: Pretell EA. Comentarios al artículo: concentraciones de yodo en orina y en sal de consumo en mujeres entre 12 a 49 años del Perú [carta]. Rev Peru Med Exp Salud Publica. 2015;32(3):611-3. 